\title{
The Soliton Correlation Matrix and the Reduction Problem for Integrable Systems *
}

\author{
J. Harnad ${ }^{1}$, Y. Saint-Aubin ${ }^{2}$, and S. Shnider ${ }^{1}$ \\ 1 Centre de recherche de mathématiques appliquées, Université de Montréal, Montréal H3C 3J7, \\ Canada, and \\ Department of Mathematics, McGill University, Montréal H3A 2T8, Canada \\ 2 Center for Theoretical Physics, Massachusetts Institute of Technology, Cambridge, MA 02139, USA
}

\begin{abstract}
Integrable $1+1$ dimensional systems associated to linear first-order matrix equations meromorphic in a complex parameter, as formulated by Zakharov, Mikhailov, and Shabat [1-3] (ZMS) are analyzed by a new method based upon the "soliton correlation matrix" ( $M$-matrix). The multi-Bäcklund transformation, which is equivalent to the introduction of an arbitrary number of poles in the ZMS dressing matrix, is expressed by a pair of matrix Riccati equations for the $M$-matrix. Through a geometrical interpretation based upon group actions on Grassman manifolds, the solution of this system is explicitly determined in terms of the solutions to the ZMS linear system. Reductions of the system corresponding to invariance under finite groups of automorphisms are also solved by reducing the $M$-matrix suitably so as to preserve the class of invariant solutions.
\end{abstract}

\section{Introduction}

Consider the pair of differential equations

$$
\left.\begin{array}{c}
\psi_{\xi}=U \psi, \\
\psi_{\eta}=V \psi,
\end{array}\right\}
$$

where $U(\lambda, \xi, \eta), V(\lambda, \xi, \eta)$, and $\psi(\lambda, \xi, \eta)$ are $n \times n$ matrix functions depending on a complex parameter $\lambda$, with $\psi$ assumed invertible, and $U$ and $V$ meromorphic in $\lambda$ with fixed poles on the Riemann sphere at $\left\{\alpha_{r}\right\}_{r=1, \ldots, l},\left\{\beta_{r}\right\}_{r=1, \ldots, m}$ respectively. Such systems were introduced and studied by Zakharov, Mikhailov, and Shabat through the use of the "dressing method" [1-3]. Expressing the $\lambda$ dependence of $U$ and $V$ explicitly as:

$$
\left.\begin{array}{l}
U=A_{0}+\sum_{r s} \frac{A_{r}^{s}}{\left(\lambda-\alpha_{r}\right)^{s}}, \\
V=B_{0}+\sum_{r s} \frac{B_{r}^{s}}{\left(\lambda-\beta_{r}\right)^{s}},
\end{array}\right\}
$$

\footnotetext{
* Supported in part by the Natural Sciences and Engineering Research Council of Canada and the "Fonds FCAC pour l'aide et le soutien à la recherche
} 
where $A_{0}, B_{0}, A_{r}^{s}, B_{r}^{s}$ are independent of $\lambda$, the integrability conditions for (1.1),

$$
U_{\eta}-V_{\xi}+[U, V]=0 \text {, }
$$

give rise to a system of nonlinear differential equations in $\left\{A_{0}, B_{0}, A_{r}^{s}, B_{r}^{s}\right\}$ which include, after applying suitable reduction procedures, many, if not all, known integrable two-dimensional system. In particular, if we restrict $U$ in (1.2) to have only simple poles at $\lambda=\infty$ and $V$ to be a polynomial in $\lambda$ and $\lambda^{-1}$ (i.e. finite order poles at 0 and $\infty$ ) and add some algebraic restrictions, then (1.1) becomes the generalized AKNS system [4], while if $A_{0}$ and $B_{0}$ are chosen to vanish and a pair of simple poles are allowed in $U$ and $V$ at -1 and +1 , respectively, we obtain the nonlinear sigma models and various other relativistically invariant 2-dimensional models by reductions [1-3, 5-8].

The "dressing method" [1-3] restricted to the generation of soliton-like additions to a given solution may be summarized as follows. Assume that a solution $\{U, V, \psi\}$ to the system (1.1) with the correct analytic structure in $\lambda$ is known. Then, following ZMS, we introduce a dressing matrix $\chi(\lambda, \xi, \eta)$ which, with its inverse $\chi^{-1}(\lambda, \xi, \eta)$, is assumed meromorphic with simple poles in $\lambda$ at fixed points $\left\{\lambda_{i}\right\}_{i=1, \ldots, K},\left\{\mu_{i}\right\}_{i=1, \ldots, K}$, respectively, and normalized such that

$$
\chi(\lambda=\infty)=\mathbb{1} \text {. }
$$

These may be expressed in terms of the residues:

$$
\begin{gathered}
Q_{i}=\frac{1}{2 \pi i} \oint_{\lambda_{i}} \chi(\lambda) d \lambda, \\
R_{i}=\frac{1}{2 \pi i} \oint_{\mu_{i}} \chi^{-1}(\lambda) d \lambda,
\end{gathered}
$$

(where the integration is on a small contour containing only the pole indicated), as :

The condition

$$
\begin{aligned}
\chi(\lambda) & =\mathbb{1}+\sum_{i=1}^{K} \frac{Q_{i}}{\lambda-\lambda_{i}}, \\
\chi^{-1}(\lambda) & =\mathbb{1}+\sum_{i=1}^{K} \frac{R_{i}}{\lambda-\mu_{i}} .
\end{aligned}
$$

$$
\chi^{-1}(\lambda) \chi(\lambda)=\mathbb{1}
$$

requires that the residues be related by the quadratic constraints:

$$
\begin{gathered}
Q_{i}+R_{i}+\sum_{\substack{j=1 \\
j \neq i}}^{K}\left[\frac{R_{i} Q_{j}}{\mu_{i}-\lambda_{j}}+\frac{R_{j} Q_{i}}{\lambda_{i}-\mu_{j}}\right]=0, \\
R_{i} Q_{i}=-\left(\mu_{i}-\lambda_{i}\right)\left[\sum_{\substack{j=1 \\
j \neq i}}^{K} \frac{R_{i} Q_{j}}{\mu_{i}-\lambda_{j}}+R_{i}\right]=\left(\mu_{i}-\lambda_{i}\right)\left[-\sum_{\substack{j=1 \\
j \neq i}}^{K} \frac{R_{j} Q_{i}}{\mu_{j}-\lambda_{i}}+Q_{i}\right] .
\end{gathered}
$$

These are expressed here in a form equally valid whether or not $\lambda_{i}=\mu_{i}$, but we assume without loss of generality that $\lambda_{i} \neq \mu_{j}$, if $i \neq j$.

A new solution $\{\tilde{U}, \tilde{V}, \tilde{\psi}\}$ to $(1.1)$ is defined by the transformation:

$$
\left.\begin{array}{l}
\Psi \rightarrow \tilde{\psi} \equiv \chi \psi, \\
U \rightarrow \tilde{U} \equiv \chi U \chi^{-1}+\chi_{\xi} \chi^{-1}, \\
V \rightarrow \tilde{V} \equiv \chi V \chi^{-1}+\chi_{\eta} \chi^{-1},
\end{array}\right\}
$$


provided the analytic structures of $\{\tilde{U}, \tilde{V}\}$ is identical to that of $\{U, V\}$; namely,

$$
\begin{aligned}
& \tilde{U}=\tilde{A}_{0}+\sum_{r, s} \frac{\tilde{A}_{r}^{s}}{\left(\lambda-\alpha_{r}\right)^{s}}, \\
& \tilde{V}=\tilde{B}_{0}+\sum_{r, s} \frac{\tilde{B}_{r}^{s}}{\left(\lambda-\beta_{r}\right)^{s}} .
\end{aligned}
$$

This implies the set of nonlinear differential equations for the residues $Q_{i}, R_{i}$ :

$$
\left.\begin{array}{l}
Q_{i \xi}=\tilde{U}\left(\lambda_{i}\right) Q_{i}-Q_{i} U\left(\lambda_{i}\right), \\
Q_{i \eta}=\tilde{V}\left(\lambda_{i}\right) Q_{i}-Q_{i} V\left(\lambda_{i}\right),
\end{array}\right\}
$$

or equivalently,

$$
\left.\begin{array}{l}
R_{i \xi}=U\left(\mu_{i}\right) R_{i}-R_{i} \tilde{U}\left(\mu_{i}\right), \\
R_{i \eta}=V\left(\mu_{i}\right) R_{i}-R_{i} \tilde{V}\left(\mu_{i}\right) .
\end{array}\right\}
$$

The nonlinearity arises from the terms $\tilde{U}, \tilde{V}$ determined by:

$$
\begin{aligned}
& \tilde{A}_{r}^{s}=\frac{1}{2 \pi i} \oint_{\alpha_{r}}\left(\lambda-\alpha_{r}\right)^{s-1} \chi(\lambda) U(\lambda) \chi^{-1}(\lambda) d \lambda, \\
& \tilde{B}_{r}^{s}=\frac{1}{2 \pi i} \oint_{\beta_{r}}\left(\lambda-\beta_{r}\right)^{s-1} \chi(\lambda) V(\lambda) \chi^{-1}(\lambda) d \lambda, \\
& \tilde{A}_{0}=A_{0}, \quad \tilde{B}_{0}=B_{0} .
\end{aligned}
$$

The system (1.11), (1.12) together with the constraints (1.8) may be regarded as a multi-Bäcklund transformation mapping the solution $\{U, V, \psi\}$ to the family of solutions $\{\tilde{U}, \tilde{V}, \tilde{\psi}\}$ determined by (1.9) after integration of (1.11), (1.11').

The purpose of the present work is the following. First, we shall present a method for integrating the system (1.11), (1.11'), (1.12) using a new quantity, the "soliton correlation matrix" or $M$-matrix, which was introduced in [8] for the case of nonlinear sigma models. All the results presented here are direct generalizations of results obtained in [8]. In the following section, we define the $M$-matrix and show that it satisfies a matrix Riccati system equivalent to (1.11), (1.12). This is explicitly solved by use of the geometrical structure of such equations based upon the action of linear groups on Grassman manifolds, as developed in [5-9]. The key step in this procedure is the recognition that the Riccati system is not the most general one associated with the given group but admits a reduction to a much smaller one, which essentially reduces the system to $2 K$ copies of Eq. (1.1), for $\lambda=\left\{\lambda_{i}\right\}$ and $\left\{\mu_{i}\right\}$.

In Sect. 3, the reduction of the systems (1.1)-(1.3) by algebraic automorphisms is studied, following the programme of Mikhailov [10,11]. We apply the reduction procedure to deduce a set of linear constraints on the $M$-matrix which are necessary and sufficient to assure that the solution generated by the multiBäcklund transformation satisfy the equations of the reduced system. As illustration of these results, the multi-soliton solutions are deduced in Sect. 4 for two specific reduced models; the nonlinear sigma model with values in $\operatorname{SL}(n, \mathbb{R}) / \operatorname{SO}(n)$ and the non-abelian two-dimensional Toda lattice. For the latter, the results reduce in the Abelian case to those obtained by Mikhailov. 


\section{The Soliton Correlation Matrix}

We define the $n K \times n K$ dimensional matrix $M$, which we call the soliton correlation matrix, such that its $i j^{\text {th }} n \times n$ block $M_{i j}$ is:

$$
M_{i j} \equiv \frac{1}{(2 \pi i)^{2}} \oint_{\mu_{\imath}} d \mu \oint_{\lambda_{j}} d \lambda \frac{\chi^{-1}(\mu) \chi(\lambda)}{\mu-\lambda}, \quad i, j=1, \ldots, k .
$$

In this definition, all choices of disjoint contours including only the poles indicated give rise to the same value for $M_{i j}$. The residues $Q_{i}, R_{i}$ and hence the dressing matrix $\chi$ and its inverse are recovered by summing along row or column blocks:

$$
Q_{i}=\sum_{j=1}^{K} M_{j i}, \quad R_{i}=-\sum_{j=1}^{K} M_{i j},
$$

as may be seen by replacing the integration on the sum of the contours at finite poles by a contour at $\infty$.

A number of further properties of the $M$-matrix following from such contour integrations will be relevant in what follows:

1. If $\mu_{i} \neq \lambda_{j}$, then

$$
M_{i j}=\frac{R_{i} Q_{i}}{\mu_{i}-\lambda_{j}}
$$

2. The constraints (1.8) on the residues are equivalent to the following ones on $M$ :

$$
\mu_{i} M_{i j}-\lambda_{j} M_{i j}=-\sum_{k l}^{K} M_{i k} M_{l j}
$$

3. There exists a block diagonal matrix $S=\operatorname{diag}\left\{s_{i}\right\}, s_{i}(\xi, \eta) \in \mathbb{C}^{n \times n}$ with $s_{i}=0$ if $\mu_{i} \neq \lambda_{i}$, such that

$$
M(D+S) M=M
$$

where $D$ is the constant matrix with $i j^{\text {th }}$ block:

$$
\left.\begin{array}{rlrl}
D_{i j} & =\frac{1}{\lambda_{i}-\mu_{j}} \mathbb{1} & \text { if } & \lambda_{i} \neq \mu_{j} \\
& =0 & \text { if } & \lambda_{i}=\mu_{j} .
\end{array}\right\}
$$

Properties 1 and 2 are immediate from the definitions and Property 3 is proved in $[8]$.

The first main result of this section is the following:

Theorem 2.1. The soliton correlation matrix $M$ satisfies the following Riccati system:

$$
\begin{aligned}
& M_{\xi}=p^{+} M-M s^{+}-M r^{+} M, \\
& M_{\eta}=p^{-} M-M s^{-}-M r^{-} M,
\end{aligned}
$$


where

$$
\left.\begin{array}{cc}
p^{+}=\operatorname{diag}\left\{U\left(\mu_{i}\right)\right\}, & p^{-}=\operatorname{diag}\left\{V\left(\mu_{i}\right)\right\}, \\
s^{+}=\operatorname{diag}\left\{U\left(\lambda_{i}\right)\right\}, & s^{-}=\operatorname{diag}\left\{V\left(\lambda_{i}\right)\right\}, \\
r_{i j}^{+}=-\frac{U\left(\mu_{j}\right)-U\left(\lambda_{i}\right)}{\mu_{j}-\lambda_{i}} & r_{i j}^{-}=-\frac{V\left(\mu_{j}\right)-V\left(\lambda_{i}\right)}{\mu_{j}-\lambda_{i}} \\
\text { if } \mu_{i} \neq \lambda_{j} & \text { if } \mu_{i} \neq \lambda_{j} \\
=-U^{\prime}\left(\mu_{i}=\lambda_{i}\right) & =-V^{\prime}\left(\mu_{i}=\lambda_{i}\right) \\
\text { if } \mu_{i}=\lambda_{i} . & \text { if } \mu_{i}=\lambda_{i} .
\end{array}\right\}
$$

This system is compatible with the constraints (2.4) and is equivalent to the multiBäcklund transformation determined by Eqs. (1.11), (1.12) with constraints (1.8).

Proof. This result generalizes one given in [8] for the case of the sigma model, and only those parts of the proof which are not immediate repetitions of those in [8] will be indicated.

Differentiating the integrand of (2.1) and using (1.9), we have

$$
\frac{\left[\chi^{-1}(\mu) \chi(\lambda)\right]_{\xi}}{\mu-\lambda}=\frac{U(\mu) \chi^{-1}(\mu) \chi(\lambda)}{\mu-\lambda}-\frac{\chi^{-1}(\mu) \chi(\lambda) U(\lambda)}{\mu-\lambda}-\chi^{-1}(\mu)\left[\frac{\tilde{U}(\mu)-\tilde{U}(\lambda)}{\mu-\lambda}\right] \chi(\lambda) .
$$

Integrating over the first two terms gives exactly the first two terms of Eq. (2.7a). The bracketed expression in the third term may be written:

$$
\begin{aligned}
\frac{\tilde{U}(\mu)-\tilde{U}(\lambda)}{\mu-\lambda} & =\frac{1}{2 \pi i}\left[\oint_{\mu} d \sigma+\oint_{\lambda} d \sigma\right] \frac{\tilde{U}(\sigma)}{(\sigma-\mu)(\sigma-\lambda)} \\
& =-\frac{1}{2 \pi i} \sum_{\alpha_{r}} \oint_{\alpha_{r}} d \sigma \frac{\tilde{U}(\sigma)}{(\sigma-\mu)(\sigma-\lambda)} \\
& =-\frac{1}{2 \pi i} \sum_{\alpha_{r}} \oint_{\alpha_{r}} d \sigma \frac{\chi(\sigma) U(\sigma) \chi^{-1}(\sigma)}{(\sigma-\mu)(\sigma-\lambda)}
\end{aligned}
$$

using Eq. (1.9) and the known location of poles in $U(\sigma)$ and $\tilde{U}(\sigma)$. Expressing $\frac{\chi(\sigma)}{\sigma-\mu}$ and $\frac{\chi^{-1}(\sigma)}{\sigma-\lambda}$ as :

$$
\begin{aligned}
\frac{\chi(\sigma)}{\sigma-\mu} & =\frac{1}{2 \pi i} \oint_{\sigma} d \alpha \frac{\chi(\alpha)}{(\alpha-\sigma)(\alpha-\mu)}=-\frac{1}{2 \pi i} \sum_{k} \oint_{\lambda_{k}} d \alpha \frac{\chi(\alpha)}{(\alpha-\sigma)(\alpha-\mu)}+\frac{\chi(\mu)}{\sigma-\mu}, \\
\frac{\chi^{-1}(\sigma)}{\sigma-\lambda} & =\frac{1}{2 \pi i} \oint_{\sigma} d \beta \frac{\chi^{-1}(\beta)}{(\beta-\sigma)(\beta-\lambda)}=-\frac{1}{2 \pi i} \sum_{l} \oint_{\mu_{l}} d \beta \frac{\chi^{-1}(\beta)}{(\beta-\sigma)(\beta-\lambda)}+\frac{\chi^{-1}(\lambda)}{\sigma-\lambda}
\end{aligned}
$$


we have:

$$
\begin{aligned}
& \oint_{\mu_{i}} d \mu \frac{\chi^{-1}(\mu) \chi(\sigma)}{(\sigma-\mu)}=\oint_{\mu_{i}} d \mu\left[-\frac{1}{2 \pi i} \sum_{k} \oint_{\lambda_{k}} d \alpha \frac{\chi^{-1}(\mu) \chi(\alpha)}{(\alpha-\sigma)(\alpha-\mu)}\right], \\
& \oint_{\lambda_{j}} d \lambda \frac{\chi^{-1}(\sigma) \chi(\lambda)}{(\sigma-\lambda)}=\oint_{\lambda_{j}} d \lambda\left[-\frac{1}{2 \pi i} \sum_{l} \oint_{\mu_{l}} d \beta \frac{\chi^{-1}(\beta) \chi(\lambda)}{(\beta-\sigma)(\beta-\lambda)}\right] .
\end{aligned}
$$

Substituting gives:

$$
\begin{aligned}
& \frac{1}{(2 \pi i)^{2}} \oint_{\mu_{i}} d \mu \oint_{\lambda_{j}} d \lambda \chi^{-1}(\mu)\left[\frac{\tilde{U}(\mu)-\tilde{U}(\lambda)}{\mu-\lambda}\right] \chi(\lambda) \\
& =\oint_{\mu_{l}} d \mu \oint_{\lambda_{j}} d \lambda \frac{1}{(2 \pi i)^{5}} \sum_{\alpha_{r}} \oint_{\alpha_{r}} d \sigma \sum_{k} \oint_{\lambda_{k}} d \alpha \\
& \quad \cdot \sum_{l} \oint_{\mu_{l}} d \beta\left[\frac{\chi^{-1}(\mu) \chi(\alpha)}{(\mu-\alpha)} \frac{U(\sigma)}{(\sigma-\alpha)(\sigma-\beta)} \frac{\chi^{-1}(\beta) \chi(\lambda)}{(\beta-\lambda)}\right] \\
& =\sum_{k l} M_{i k} \frac{1}{2 \pi i} \sum_{\alpha_{r}} \oint_{\alpha_{r}} d \sigma\left[\frac{U(\sigma)}{\left(\sigma-\lambda_{k}\right)\left(\sigma-\mu_{l}\right)}\right] M_{l j} \\
& =\sum_{k l} M_{i k} r_{k l}^{+} M_{l j},
\end{aligned}
$$

where $r_{k l}^{+}$is as defined in Eq. (2.8). A similar computation yields (2.7b) for the $\eta$-derivative.

The compatibility of Eqs. (2.7a) and (2.7b) with the constraints (2.4) follows most easily from the geometrical interpretation of matrix Riccati systems which we summarize briefly here. (A more detailed account is given in $[6,8]$.) Introduce a $\mathrm{GL}(n K, \mathbb{C})$-valued function $\mathscr{G}(\xi, \eta)$ determined by the system of linear equations:

$$
\begin{aligned}
\mathscr{G}_{\xi} & =\left(\begin{array}{ll}
p^{+} & 0 \\
r^{+} & s^{+}
\end{array}\right) \mathscr{G}, \\
\mathscr{G}_{\eta} & =\left(\begin{array}{cc}
p^{-} & 0 \\
r^{-} & s^{-}
\end{array}\right) \mathscr{G},
\end{aligned}
$$

with some suitable chosen initial value $\mathscr{G}\left(\xi_{0}, \eta_{0}\right)$. The solution will be of block triangular form

$$
\mathscr{G}=\left(\begin{array}{ll}
P & 0 \\
R & S
\end{array}\right),
$$

provided $\mathscr{G}\left(\xi_{0}, \eta_{0}\right)$ is. The integrability condition for (2.9a) and (2.9b) is identical to that for (2.7a) and (2.7b), namely,

$$
\left(\begin{array}{ll}
p_{+} & 0 \\
r_{+} & s_{+}
\end{array}\right)_{\eta}-\left(\begin{array}{ll}
p_{-} & 0 \\
r_{-} & s_{-}
\end{array}\right)_{\xi}+\left[\left(\begin{array}{ll}
p_{+} & 0 \\
r_{+} & s_{+}
\end{array}\right),\left(\begin{array}{ll}
p_{-} & 0 \\
r_{-} & s_{-}
\end{array}\right)\right]=0,
$$

which is equivalent to Eq. (1.3) evaluated at $\lambda=\left\{\lambda_{i}, \mu_{i}\right\}$. The general solution to Eq. (2.7a) and (2.7b) is given in terms of $\mathscr{G}$ by the linear fractional transformation

$$
M(\xi, \eta)=P(\xi, \eta) m[R(\xi, \eta) m+S(\xi, \eta)]^{-1},
$$


where $m$ is an arbitrary constant $2 n K \times 2 n K$ dimensional matrix satisfying the constraints (2.4). The geometrical significance of this is that $M(\xi, \eta)$ may be regarded as the affine coordinates of a point in the complex Grassman manifold $G_{n K}(2 n K, \mathbb{C})$ of $n K$-planes in $\mathbb{C}^{2 n K}$. The group $\mathrm{GL}(2 n K, \mathbb{C})$ acts on $G_{n K}(2 n K, \mathbb{C})$, in a natural manner which, expressed in affine coordinates is given by linear fractional transformations

$$
\begin{gathered}
\left(\begin{array}{ll}
P & Q \\
R & S
\end{array}\right): m \rightarrow(P m+Q)(R m+S)^{-1}, \\
\left(\begin{array}{ll}
P & Q \\
R & S
\end{array}\right) \in \operatorname{GL}(2 n K, \mathbb{C}), \quad m \in \mathbb{C}^{n K \times n K} .
\end{gathered}
$$

(For the action to be effective, the group must be quotiented by its center.) The function $M: \mathbb{R}^{2} \rightarrow \mathbb{C}^{n K \times n K}$ may be regarded as defining a section of the trivial Grassmannian bundle $\mathbb{R}^{2} \times G_{n K}\left(\mathbb{C}^{2 n K}\right)$, and similarly $\mathscr{G}: \mathbb{R}^{2} \rightarrow \mathrm{GL}(2 n K, \mathbb{C})$ defines a section of the trivial principal bundle $\mathbb{R}^{2} \times \mathrm{GL}(2 n K, \mathbb{C})$. The Eqs. $(2.7 \mathrm{a})$ and $(2.7 \mathrm{~b})$ and $(2.8 \mathrm{a})$ and $(2.8 \mathrm{~b})$ express the fact that these sections are covariant constant with respect to the integrable connection defined by the $\operatorname{gl}(2 n K, \mathbb{C})$ valued form:

$$
\tilde{\omega}_{(\xi, \eta, \mathscr{G})}=A d \mathscr{G}^{-1} \omega+\mathscr{G}^{-1} d \mathscr{G},
$$

where

$$
\omega=-\left(\begin{array}{ll}
p_{+} & 0 \\
r_{+} & s_{+}
\end{array}\right) d \xi-\left(\begin{array}{ll}
p_{-} & 0 \\
r_{-} & s_{-}
\end{array}\right) d \eta,
$$

and the integrability condition (2.11) expresses the vanishing of the curvature. The constraint (2.4) expresses the fact that $M$ has values in the submanifold of fixed points under the map: $T: G_{n K}\left(\mathbb{C}^{2 n K}\right) \rightarrow G_{n K}\left(\mathbb{C}^{2 n K}\right)$, defined by the matrix :

$$
\begin{gathered}
T=\tau \otimes \mathbb{1}_{n \times n}, \\
\tau=\left(\begin{array}{c:c}
\operatorname{diag}\left\{\mu_{i}\right\} & 0 \\
\hdashline-E & \operatorname{diag}\left\{\bar{\lambda}_{i}\right\}
\end{array}\right), \\
E=\left(\begin{array}{ccc}
1 & \ldots & 1 \\
\vdots & & \vdots \\
1 & \ldots & 1
\end{array}\right)
\end{gathered}
$$

acting, as in Eq. (2.12) by linear fractional transformations. This constraint is consistent with Eqs. (2.7a) and (2.7b) because the connection form commutes with $T:[\omega, T]=0$, and hence the infinitesimal holonomy group is contained in the subgroup of $\mathrm{GL}(2 n K, \mathbb{C})$ of elements commuting with $T$.

The solution to Eqs. (2.9a) and (2.9b) may be explicitly given in terms of the solutions of Eq. (1.1) by noting that this subgroup reduction leads to a block diagonalization of the connection form by a transformation independent of $(\xi, \eta)$. The specific form of the subgroup reduction depends on whether degeneracies occur between the constants $\left\{\lambda_{i}, \mu_{i}\right\}$ which are the eigenvalues of the matrix $\tau$. Since we may assume without loss of generality that the $\left\{\lambda_{i}\right\}$ and $\left\{\mu_{i}\right\}$ are 
separately distinct and the only possible degeneracies occur between pairs $\left\{\lambda_{i}, \mu_{i}\right\}$, the Jordan blocks are all 1- and 2-dimensional. The matrix of generalized eigenvectors is :

$$
S=\left(\begin{array}{ll}
\mathbb{1} & 0 \\
d & \mathbb{1}
\end{array}\right),
$$

where

$$
\left.\begin{array}{lll}
d_{i j}=\frac{1}{\lambda_{i}-\mu_{j}} & \text { if } & \lambda_{i} \neq \mu_{j} . \\
d_{i i}=0 & \text { if } & \lambda_{i}=\mu_{i} .
\end{array}\right\}
$$

The simultaneous block diagonalization of $T$ and $\omega$ by the matrix $S \otimes \mathbb{1}$ leads to the solution of (2.9a) and (2.9b) in the form:

$$
\mathscr{G}=S \otimes \mathbb{1} \mathscr{G}_{0} S^{-1} \otimes \mathbb{1}
$$

where $\mathscr{G}_{0}$ satisfies:

$$
\begin{aligned}
\mathscr{G}_{0, \xi} & =\left(\begin{array}{c:c}
\tilde{U} & 0 \\
\hdashline \hat{U} & U
\end{array}\right) \mathscr{G}_{0}, \\
\mathscr{G}_{0, \eta} & =\left(\begin{array}{c:c}
\tilde{V} & 0 \\
\hdashline \hat{V} & V
\end{array}\right) \mathscr{G}_{0},
\end{aligned}
$$

with the block diagonal matrices $\tilde{U}, U, \hat{U}, \tilde{V}, V, \hat{V}$ given by:

$$
\begin{aligned}
& \tilde{U}=\operatorname{diag}\left\{U\left(\mu_{i}\right)\right\}, \\
& U=\operatorname{diag}\left\{U\left(\lambda_{i}\right)\right\}, \\
& \tilde{V}=\operatorname{diag}\left\{V\left(\mu_{i}\right)\right\}, \\
& V=\operatorname{diag}\left\{V\left(\lambda_{i}\right)\right\}, \\
& \hat{U}=\operatorname{diag}\left\{U_{i}\right\}, \\
& \hat{V}=\operatorname{diag}\left\{V_{i}\right\},
\end{aligned}
$$

where

$$
\begin{aligned}
U_{i} & =-U^{\prime}\left(\lambda_{i}\right) & & \text { if } \quad \lambda_{i}=\mu_{i} \\
& =0 & & \text { otherwise } \\
V_{i} & =-V^{\prime}\left(\lambda_{i}\right) & & \text { if } \quad \lambda_{i}=\mu_{i} \\
& =0 & & \text { otherwise }
\end{aligned}
$$

If there are no degeneracies and $\mathscr{G}_{0}\left(\xi_{0}, \eta_{0}\right)$ is taken as $n \times n$ block diagonal, Eqs. (2.19a) and (2.19b) is just $2 K$ copies of (1.1) evaluated at $\lambda=\left\{\lambda_{i}, \mu_{i}\right\}$. If $\lambda_{i}=\mu_{i}$ for certain $i$, the corresponding blocks reduce to the solution of the block triangular 
$2 n \times 2 n$ dimensional system

$$
\begin{aligned}
& \left(\begin{array}{ll}
\psi & 0 \\
\phi & \psi
\end{array}\right)_{\xi}=\left(\begin{array}{rr}
U(\lambda) & 0 \\
-U^{\prime}(\lambda) & U(\lambda)
\end{array}\right)\left(\begin{array}{ll}
\psi & 0 \\
\phi & \psi
\end{array}\right), \\
& \left(\begin{array}{ll}
\psi & 0 \\
\phi & \psi
\end{array}\right)_{\eta}=\left(\begin{array}{rr}
V(\lambda) & 0 \\
-V^{\prime}(\lambda) & V(\lambda)
\end{array}\right)\left(\begin{array}{ll}
\psi & 0 \\
\phi & \psi
\end{array}\right),
\end{aligned}
$$

whose general solution is determined by a solution $\psi$ of (1.1), plus a solution to the equation obtained from (1.1) by differentiating with respect to $\lambda$, namely:

$$
\phi(\lambda)=-\psi^{\prime}(\lambda)+\psi(\lambda) C(\lambda),
$$

where $C(\lambda)$ is an arbitrary $n \times n$ matrix constant in $(\xi, \eta)$.

Combining these results, we find the general solution of (2.9a) and (2.9b) to be of the form

$$
\mathscr{G}=\left(\begin{array}{cc}
\tilde{\Psi} & 0 \\
D \tilde{\Psi}-\Psi D+\Phi & \Psi
\end{array}\right)
$$

where

$$
\left.\begin{array}{l}
\tilde{\Psi}=\operatorname{diag}\left\{\psi\left(\mu_{i}\right)\right\}, \\
\Psi=\operatorname{diag}\left\{\psi\left(\lambda_{i}\right)\right\}, \\
\Phi=\operatorname{diag}\left\{\phi_{i}\right\}
\end{array}\right\}
$$

with

$$
\begin{aligned}
& \phi_{i}=-\psi^{\prime}\left(\lambda_{i}\right)+\psi\left(\lambda_{i}\right) C\left(\lambda_{i}\right) \quad \text { if } \quad \lambda_{i}=\mu_{i} \\
& =0 \quad \text { if } \lambda_{i} \neq \mu_{i}
\end{aligned}
$$

and $D$ as defined in Eq. (2.6). Combining with Eq. (2.12), we arrive at the following result.

Theorem 2.2. The general solution of the system (2.7a) and (2.7b) is :

$$
M=\tilde{\Psi} m[(D \tilde{\Psi}-\Psi D+\Phi) m+\Psi]^{-1},
$$

where $\Psi, \tilde{\Psi}$, and $\Phi$ are defined in Eq. (2.24).

This form of the solution is identical for all integrable systems of type (1.1)-(1.3) and was explicitly given in [8] for the nonlinear $\sigma$-model. Another form of the solution given there relates the result to the notation of Zakharov, Mikhailov, and Shabat and we quote it here for completeness. The proof for the general case coincides with that given in [8] and will not be repeated.

Theorem 2.3. The solution (2.25) of Theorem 2.2 is equivalently expressed in terms of the residues $\left\{Q_{i}, R_{i}\right\}$ defining the ZMS dressing matrix $\chi$ and its inverse as follows:

$$
\left.\begin{array}{l}
Q_{i}=X_{i} F_{i}^{+}, \\
R_{i}=H_{i} K_{i}^{+}
\end{array}\right\}
$$


where $\left\{X_{i}, F_{i}\right\}$ and $\left\{H_{i}, K_{i}\right\}$ are pairs of rectangular maximal rank matrices of dimension $n \times q_{i}$ and $n \times r_{i}$ respectively, with $F_{i}, H_{i}$ determined by

$$
\left.\begin{array}{l}
F_{i}=\psi^{\dagger-1}\left(\lambda_{i}\right) f_{i}, \\
H_{i}=\psi\left(\mu_{i}\right) h_{i},
\end{array}\right\}
$$

in terms of arbitrary constant maximal rank matrices $f_{i} \in \mathbb{C}^{n \times q_{i}}, h_{i} \in \mathbb{C}^{n \times r_{i}}$, and $X_{i}, K_{i}$ are determined by solving the linear system:

and

$$
\left.\begin{array}{l}
\sum_{i} X_{i} \Gamma_{i j}=H_{j} \\
\sum_{i} K_{i} \Gamma_{i j}^{+}=F_{j}
\end{array}\right\}
$$

$$
\begin{gathered}
\Gamma_{i j}=\frac{F_{i}^{+} H_{j}}{\lambda_{i}-\mu_{j}} \quad \text { if } \quad \lambda_{i} \neq \mu_{j}, \\
\Gamma_{i i}=F_{i}^{+} \phi_{i} \psi^{-1}\left(\lambda_{i}\right) H_{i} \quad \text { if } \quad \lambda_{i}=\mu_{i},
\end{gathered}
$$

with

$$
F_{i}^{+} H_{i}=0
$$

in the latter case.

This completes the analysis for the general case. In the next section we shall show how these results may be applied to the solution of the system (1.1)-(1.3) reduced by suitable algebraic constraints.

\section{Algebraic Reductions}

Following the programme suggested by Mikhailov $[10,11]$, we now consider reduced systems obtained from (1.1) by the application of certain additional constraints corresponding to invariance under finite automorphism groups. The transformations leaving (1.1) invariant which will be considered are of the type:

$$
\left.\begin{array}{l}
\psi(\lambda) \rightarrow f \sigma\left[\psi\left(\widetilde{s^{-1}(\lambda)}\right)\right] \equiv \hat{\psi}(\lambda), \\
U(\lambda) \rightarrow f \sigma_{*} U\left(\widetilde{s^{-1}(\lambda)}\right) f^{-1}+f_{\xi} f^{-1} \equiv \hat{U}(\lambda), \\
V(\lambda) \rightarrow f \sigma_{*} V\left(\widetilde{s^{-1}(\lambda)}\right) f^{-1}+f_{\eta} f^{-1} \equiv \hat{V}(\lambda),
\end{array}\right\}
$$

where $s: \mathbb{C} P^{1} \rightarrow \mathbb{C} P^{1}$ is a conformal map of the Riemann sphere, $\sigma \in \operatorname{Aut}[\mathrm{GL}(n, \mathbb{C})]$, $f \in C^{\infty}\left(\mathbb{R}^{2}, \mathrm{GL}(n, \mathbb{C})\right)$, and $\tilde{\lambda}=\lambda$ if $\sigma_{*}$ is linear, $\tilde{\lambda}=\bar{\lambda}$ if $\sigma_{*}$ is anti-linear. We may, up to a sign, define $s$ as an element of $\operatorname{SL}(2, \mathbb{C})$

$$
s=\left(\begin{array}{ll}
a & b \\
c & d
\end{array}\right), \quad a d-b c=1,
$$

acting on the complex plane by linear fractional transformations

$$
s: \lambda \rightarrow \frac{a \lambda+b}{c \lambda+d}
$$


and preserving the set of poles $\left\{\alpha_{r}\right\}$ and $\left\{\beta_{r}\right\}$. The automorphisms of $\operatorname{GL}(n, \mathbb{C})$ are one of the following types:

$$
\begin{aligned}
& \sigma_{1}(g)=\operatorname{tg} t^{-1}, \\
& \sigma_{2}(g)=\operatorname{tg} t^{-1}, \\
& \sigma_{3}(g)=\operatorname{tg}^{T-1} t^{-1}, \\
& \sigma_{4}(g)=\operatorname{tg}^{\dagger-1} t^{-1},
\end{aligned}
$$

where $t$ is some fixed element of $\operatorname{GL}(n, \mathbb{C})$. The function $f(\xi, \eta)$ defines a gauge transformation and may be thought of as determining the normalization at $\lambda=\infty$. In particular, if we choose the canonical gauge:

$$
U(\infty)=\tilde{U}(\infty)=0, \quad V(\infty)=\tilde{V}(\infty)=0,
$$

then $f$ is uniquely determined (up to a constant) as

$$
f=\sigma\left[\psi\left(\overline{\left(s^{-1}(\infty)\right.}\right]^{-1} .\right.
$$

In general, we shall consider a finite group $\left\{f_{\alpha}, \sigma_{\alpha}, s_{\alpha}\right\}_{\alpha=1, \ldots, p}$ of such transformations, which means that the gauge transformations

$$
f=f(\sigma, s)
$$

must satisfy the composition rule:

$$
f\left(\sigma_{\beta}, \sigma_{\alpha}, s_{\beta}, s_{\alpha}\right)=f\left(\sigma_{\beta}, s_{\beta}\right) \sigma_{\beta} f\left(\sigma_{\alpha}, s_{\alpha}\right),
$$

and the poles $\left\{\alpha_{r}\right\}$ and $\left\{\beta_{r}\right\}$ must each be the union of a set of orbits of the finite subgroup $\left\{s_{\alpha}\right\} \subset \operatorname{SL}(2, \mathbb{C})$ in $\mathbb{C} P^{1}$.

We now consider the solutions of (1.1) which are invariant under the transformations (3.1) for all elements of this group. The invariance conditions

$$
\begin{aligned}
& \psi(s(\tilde{\lambda}))=f \sigma \psi(\lambda), \\
& U(s(\tilde{\lambda}))=f \sigma_{*}[U(\lambda)] f^{-1}+f_{\xi} f^{-1}, \\
& V(s(\tilde{\lambda}))=f \sigma_{*}[V(\lambda)] f^{-1}+f_{\eta} f^{-1},
\end{aligned}
$$

imply in particular that $f$ is not arbitrary but determined by evaluating (3.9a) at one point, which we choose as $\lambda=\infty$, giving

$$
f=\psi(\infty) \sigma\left[\psi\left(\widetilde{s^{-1}(\infty)}\right)\right]^{-1} .
$$

The composition rule (3.8) for various $\left\{\sigma_{\alpha}, s_{\alpha}\right\}$ is implied by the invariance condition (3.9a). Conditions (3.9b) and (3.9c) are equivalent to the following relations for the matrix functions $\left\{A_{r}^{s}, B_{r}^{s}\right\}$, defining the reduced system

$$
\begin{aligned}
& \sigma_{*}\left(A_{r}^{t}\right)=\left(c \tilde{\alpha}_{r}+d\right)^{2 t} \sum_{q=0} \frac{(q+t) !}{q ! t !} c^{q}\left(c \tilde{\alpha}_{r}+d\right)^{q} f^{-1} A_{s\left(\tilde{\alpha}_{r}\right)}^{q+t} f, \\
& \sigma_{*}\left(B_{r}^{t}\right)=\left(c \tilde{\beta}_{r}+d\right)^{2 t} \sum_{q=0} \frac{(q+t) !}{q ! t !} c^{q}\left(c \tilde{\beta}_{r}+d\right)^{q} f^{-1} B_{s\left(\tilde{\beta}_{r}\right)}^{q+t} f .
\end{aligned}
$$


These relations are most easily derived by evaluating $\left\{A_{r}^{s}, B_{r}^{s}\right\}$ by the contour integrals :

$$
\begin{aligned}
& A_{r}^{s}=\frac{1}{2 \pi i} \oint_{\alpha_{r}}\left(\lambda-\alpha_{r}\right)^{s-1} U(\lambda) d \lambda, \\
& B_{r}^{s}=\frac{1}{2 \pi i} \oint_{\beta_{r}}\left(\lambda-\beta_{r}\right)^{s-1} V(\lambda) d \lambda,
\end{aligned}
$$

applied to both sides of (3.9b) and (3.9c). No further relation on $A_{0}, B_{0}$ is implied since the $\lambda \rightarrow \infty$ limit of (3.9b) and (3.9c) is already satisfied by the choice (3.10) for $f$.

If we now require that under the transformation (1.9), both the old and new solutions $\{U, V, \psi\},\{\tilde{U}, \tilde{V}, \tilde{\psi}\}$ satisfy constraints of the type (3.9), we deduce that the dressing matrix $\chi$ and its inverse must satisfy:

$$
\sigma[\chi(\lambda)]=\tilde{f}^{-1} \chi(s(\tilde{\lambda})) f
$$

where $\tilde{f}$ is determined in terms of $\tilde{\psi}$ by a relation of the same form as (3.6). This requires in particular that the set of poles $\left\{\lambda_{i}, \mu_{i}\right\}$ be invariant under $s: \lambda \rightarrow s(\tilde{\lambda})$ with the sets $\left\{\lambda_{i}\right\}$ and $\left\{\mu_{i}\right\}$ separately invariant for automorphisms of type $\sigma_{1}$ and $\sigma_{2}$ and mapped into each other for those of type $\sigma_{3}$ and $\sigma_{4}$. The following theorem gives the constraints on the soliton correlation matrix which assure that the new solution $\{\tilde{U}, \tilde{V}, \tilde{\psi}\}$ determined by Theorem 2.2 satisfies the constraints (3.9), provided $\{U, V, \psi\}$ does.

Theorem 3.1. Suppose $\{U, V, \psi\}$ is a solution of (1.1) satisfying the invariance conditions (3.9) for an automorphism $(\sigma, s)$ with gauge transformation $f$. The new solution $\{\tilde{U}, \tilde{V}, \tilde{\psi}\}$ determined by the multi-Bäcklund transformation (1.9) with dressing matrix $\chi$ given by Theorem 2.2 and Eqs. (1.6), (2.2) satisfies the corresponding invariance conditions (3.9) with gauge transformation $f$ if and only if the $M$-matrix satisfies one of the following set of constraints:

(1) If $\sigma$ is of type $\sigma_{1}$,

$$
M_{s(i) s(j)}=\frac{f t M_{i j}(f t)^{-1}}{\left(c \mu_{i}+d\right)\left(c \lambda_{j}+d\right)} .
$$

(2) If $\sigma$ is of type $\sigma_{2}$,

$$
M_{s(\bar{i}) s(\bar{j})}=\frac{(f t) \bar{M}_{i j}(f t)^{-1}}{\left(c \bar{\mu}_{i}+d\right)\left(c \bar{\lambda}_{j}+d\right)} .
$$

(3) If $\sigma$ is of type $\sigma_{3}$,

$$
M_{s(j) s(i)}=\frac{-f t M_{i j}^{T}(f t)^{-1}}{\left(c \mu_{i}+d\right)\left(c \lambda_{j}+d\right)} .
$$

(4) If $\sigma$ is of type $\sigma_{4}$,

$$
M_{s(\bar{j}) s(\bar{i})}=\frac{-f t M_{i j}^{\dagger}(f t)^{-1}}{\left(c \bar{\mu}_{i}+d\right)\left(c \bar{\lambda}_{j}+d\right)}
$$


where

$$
M_{s(\tilde{i}) s(\tilde{j})} \equiv \frac{1}{(2 \pi i)^{2}} \oint_{s\left(\tilde{\mu}_{1}\right)} d \mu \oint_{s\left(\tilde{\lambda}_{J}\right)} d \lambda \frac{\chi^{-1}(\mu) \chi(\lambda)}{\mu-\lambda}
$$

for $\sigma_{1}$ and $\sigma_{2}$, and

$$
M_{s(\tilde{j}) s(\tilde{i})} \equiv \frac{1}{(2 \pi i)^{2}} \oint_{s\left(\tilde{\lambda}_{j}\right)} d \mu \oint_{s\left(\tilde{\mu}_{i}\right)} d \lambda \frac{\chi^{-1}(\mu) \chi(\lambda)}{\mu-\lambda}
$$

for $\sigma_{3}$ and $\sigma_{4}$.

Proof. This is a direct generalization of Theorem 5.1 of [8] which is proved there in detail, therefore we only give an outline here. From the meromorphic structure of $\chi(\lambda)$ and $\chi^{-1}(\mu)$ and their behaviour as $\lambda, \mu \rightarrow \infty$, the constraint (3.13) is equivalent to:

$$
\oint_{\mu_{i}} d \mu \oint_{\lambda_{j}} d \lambda \frac{\sigma\left[\chi^{-1}(\mu) \chi(\lambda)\right]}{\mu-\lambda}=f^{-1} \oint_{\mu_{i}} d \mu \oint_{\lambda_{j}} d \lambda \frac{\chi^{-1}(s(\tilde{\mu})) \chi(s(\tilde{\lambda}))}{\mu-\lambda} f .
$$

Consider case (1). Since $\sigma$ is now linear, we have, by the definition (2.1) of $M_{i j}$,

$$
\sigma\left(M_{i j}\right)=\frac{1}{(2 \pi i)^{2}} \oint_{\mu_{\imath}} d \mu \oint_{\lambda_{j}} d \lambda \frac{\sigma\left[\chi^{-1}(\mu) \chi(\lambda)\right]}{\mu-\lambda} .
$$

Changing variables to $\mu^{\prime}=s(\mu), \lambda^{\prime}=s(\lambda)$ and integrating around $s\left(\mu_{i}\right), s\left(\lambda_{j}\right)$ gives the relation (3.14a). A similar substitution taking complex conjugation into account yields (3.14b) for case (2). For case (3), $\sigma$ is not linear, but defining the linear antiautomorphism $\hat{\sigma}(g)=\sigma\left(g^{-1}\right)=t g^{T} t^{-1}$, we have

$$
\begin{aligned}
\hat{\sigma}\left(M_{i j}\right) & =\frac{1}{(2 \pi i)^{2}} \oint_{\mu_{2}} d \mu \oint_{\lambda_{J}} d \lambda \frac{\hat{\sigma}\left[\chi^{-1}(\mu) \chi(\lambda)\right]}{\mu-\lambda} \\
& =\frac{1}{(2 \pi i)^{2}} \oint_{\mu_{i}} d \mu \oint_{\lambda_{J}} d \lambda \frac{\sigma\left[\chi^{-1}(\lambda) \chi(\mu)\right]}{\mu-\lambda} \\
& =\frac{1}{(2 \pi i)^{2}} f^{-1} \oint_{\mu_{i}} d \mu \oint_{\lambda_{J}} d \lambda \frac{\chi^{-1}(s(\lambda)) \chi(s(\mu))}{\mu-\lambda} f .
\end{aligned}
$$

Now changing variables to $\mu^{\prime}=s(\lambda), \lambda^{\prime}=s(\mu)$ and integrating around $s\left(\lambda_{j}\right)$ and $s\left(\mu_{i}\right)$ gives the relation (3.14c) and a similar computation with complex conjugates gives (3.14d).

The constraints (3.14a)-(3.14d) all have a natural interpretation in terms of the Grassmannian bundles $\mathbb{R}^{2} \times G_{n K}\left(\mathbb{C}^{2 n K}\right)$, and this interpretation can be used to prove the consistency of these constraints with Eqs. (2.7a) and (2.7b). Define first four $k$-dimensional representations $R, \tilde{R}, Q$, and $\tilde{Q}$ of the group $\left\{s_{\alpha}\right\} \subset \operatorname{SL}(2, \mathbb{C})$ with matrix elements

$$
\begin{array}{ll}
R_{i j}(s)=\frac{\delta_{i, s(j)}}{c \mu_{j}+d}, & \tilde{R}_{i j}(s)=\frac{\delta_{i, s(\bar{j})}}{c \bar{\mu}_{j}+d}, \\
Q_{i j}(s)=\delta_{i, s(j)}\left(c \lambda_{j}+d\right), & \tilde{Q}_{i j}(s)=\delta_{i, s(\bar{j})}\left(c \bar{\lambda}_{j}+d\right) .
\end{array}
$$


In terms of these matrices, the four conditions (3.14a)-(3.14d) may be expressed:

(1) For $\sigma$ of type $\sigma_{1}$,

$$
(R \otimes f t) M=M(Q \otimes f t) .
$$

(2) For $\sigma$ of type $\sigma_{2}$,

$$
(\tilde{R} \otimes f t) \bar{M}=M(\tilde{Q} \otimes f t) .
$$

(3) For $\sigma$ of type $\sigma_{3}$,

$$
\left(R \otimes(f t)^{T-1}\right) M+M^{T}\left(Q \otimes(f t)^{T-1}\right)=0 .
$$

(4) For $\sigma$ of type $\sigma_{4}$,

$$
\left(\tilde{\tilde{R}} \otimes(f t)^{\dagger-1}\right) M+M^{\dagger}\left(\tilde{\tilde{Q}} \otimes(f t)^{\dagger-1}\right)=0,
$$

where in each case $f=f(\sigma, s)$ and $t$ is the $\operatorname{GL}(n, \mathbb{C})$ element appearing in the definition (3.4) of $\sigma$. These conditions are interpreted geometrically by defining for each $f$ the four cases (1)-(4), a function on $\mathbb{R}^{2}$ with values, respectively, in the space of linear maps, anti-linear maps, bilinear and sesquilinear forms on $\mathbb{C}^{2 n K}$ :

1) For $\sigma_{1}$, the linear map $L(\xi, \eta): \mathbb{C}^{2 n K} \rightarrow \mathbb{C}^{2 n K}$ with matrix representation,

$$
L X=\left(\begin{array}{ll}
R & 0 \\
0 & Q
\end{array}\right) \otimes f t X .
$$

2) For $\sigma_{2}$, the anti-linear map $\hat{L}(\xi, \eta): \mathbb{C}^{2 n K} \rightarrow \mathbb{C}^{2 n K}$ with matrix representation,

$$
\tilde{L} X=\left(\begin{array}{cc}
\tilde{R} & 0 \\
0 & \tilde{Q}
\end{array}\right) \otimes f t \bar{X}
$$

3) For $\sigma_{3}$, the bilinear form $B(\xi, \eta): \mathbb{C}^{2 n K} \times \mathbb{C}^{2 n K} \rightarrow \mathbb{C}$ with matrix representation,

$$
B(X, Y)=X^{T}\left(\begin{array}{ll}
0 & Q \\
R & 0
\end{array}\right) \otimes(f t)^{T-1} Y .
$$

4) For $\sigma_{4}$, the sesquilinear form: $S(\xi, \eta): \mathbb{C}^{2 n K} \times \mathbb{C}^{2 n K} \rightarrow \mathbb{C}^{2 n K}$ with matrix representation,

$$
S(X, Y)=X^{\dagger}\left(\begin{array}{cc}
0 & \tilde{Q} \\
\tilde{\tilde{R}} & 0
\end{array}\right) \otimes(f t)^{\dagger-1} Y
$$

The maps $L$ and $\tilde{L}$ act naturally on the Grassmann manifold $G_{n K}\left(\mathbb{C}^{2 n K}\right)$, and at each point $(\xi, \eta)$ the constraints $(3.17 \mathrm{a})$ and $(3.17 \mathrm{~b})$ are the expressions in affine coordinates of the fact that the corresponding point in $G_{n K}\left(\mathbb{C}^{2 n K}\right)$ is fixed under them:

$$
\begin{aligned}
& L\left[\begin{array}{c}
M \\
\mathbb{1}
\end{array}\right]=\left[\begin{array}{c}
M \\
\mathbb{1}
\end{array}\right], \\
& \tilde{L}\left[\begin{array}{c}
M \\
\mathbb{1}
\end{array}\right]=\left[\begin{array}{c}
M \\
\mathbb{1}
\end{array}\right],
\end{aligned}
$$


where $\left[\begin{array}{c}M \\ \mathbb{1}\end{array}\right]$ denotes the equivalence class of all $2 n K \times 2 n K$ dimensional rectangular matrices $\left(\begin{array}{c}M G \\ G\end{array}\right), G \in \mathrm{GL}(n K, \mathbb{C})$ with columns spanning the corresponding $n K$ plane. Similarly, the constraints (3.17c) and (3.17d) express the fact that for all $(\xi, \eta),\left[\begin{array}{c}M \\ \mathbb{1}\end{array}\right]$ determines an $n K$-plane which is totally isotropic under $B$ or $S$, respectively:

$$
\begin{aligned}
& {\left[\begin{array}{ll}
M^{T} & \mathbb{1}
\end{array}\right] B\left[\begin{array}{c}
M \\
\mathbb{1}
\end{array}\right]=0,} \\
& {\left[\begin{array}{ll}
M^{\dagger} & \mathbb{1}
\end{array}\right] S\left[\begin{array}{c}
M \\
\mathbb{1}
\end{array}\right]=0 .}
\end{aligned}
$$

These constraints may be restated in terms of the Grassmannian bundle $\mathbb{R}^{2} \times G_{n K}\left(\mathbb{C}^{2 n K}\right)$ of which $M(\xi, \eta)$ determines a covariant constant section. The quantities $L, \tilde{L}, B$, and $S$ may at each $(\xi, \eta)$ be regarded as tensors on the fibre of the trivial vector bundle $\mathbb{R}^{2} \times \mathbb{C}^{2 n K}$ over that point. Thus $L, \tilde{L}, B$, and $S$ define sections of the corresponding tensorial bundles associated to the principal bundle $\mathbb{R}^{2} \times G(2 n K, \mathbb{C})$ by the group actions :

$$
\begin{gathered}
\mathscr{G}: L \rightarrow \mathscr{G} L \mathscr{G}^{-1}, \\
\mathscr{G}: \tilde{L} \rightarrow \mathscr{G} \tilde{L} \mathscr{G}^{-1}, \\
\mathscr{G}: B \rightarrow \mathscr{G}^{T-1} B \mathscr{G}^{-1}, \\
\mathscr{G}: S \rightarrow \mathscr{G}^{\dagger-1} S \mathscr{G}^{-1} .
\end{gathered}
$$

(In fact, the sections $L, \tilde{L}$ associated to the various group elements $\left\{\sigma_{\alpha}, s_{\alpha}\right\}$ define a representation of the reduction group.) The constraints $(3.17 \mathrm{a})-(3.17 \mathrm{~d})$ define reductions of the Grassmannian bundle $\mathbb{R}^{2} \times G_{n K}\left(\mathbb{C}^{2 n K}\right)$ to sub-bundles consisting of fixed points under $L$ or $\tilde{L}$ or isotropic subspaces under $B$ or $S$, respectively. Since the section determined by $M(\xi, \eta)$ is covariant constant, the consistency of the constraints (3.17a)-(3.17d) with Eqs. (2.7a) and (2.7b) follows provided the sections determined by $L, \tilde{L}, B$, and $S$ are themselves covariant constant. This is the constant of the next theorem.

Theorem 3.2. The functions $L, \tilde{L}, B$, and $S$ satisfy the covariant constancy conditions

$$
\begin{aligned}
& d)+[\omega, L]=0, \\
& \text { 2) } \\
& \text { 3) } \\
& \text { 4) } \\
& d B-\omega \tilde{L}-\omega^{T} B-B \omega=0, \\
& d S-\omega^{\dagger} S-S \omega=0,
\end{aligned}
$$

where $\omega$ is defined by Eq. (2.14), and therefore the constraints (3.17a)-(3.17d) are consistent with Eqs. (2.7a) and (2.7b) determining $M$. 
Proof. The $\xi$ component of Eq. (3.21a) is

$$
\left(\begin{array}{cc}
R & 0 \\
0 & Q
\end{array}\right) \otimes f_{\xi} t-\left[\left(\begin{array}{cc}
p^{+} & 0 \\
r^{+} & s^{+}
\end{array}\right),\left(\begin{array}{cc}
R & 0 \\
0 & Q
\end{array}\right) \otimes f t\right],
$$

where $p^{+}, r^{+}$, and $s^{+}$are defined in Eq. (2.8). From the definition (3.10) of $R$ and $Q$, it follows that the lower triangular block $\left(\begin{array}{ll}0 & 0 \\ \mathrm{r}^{+} & 0\end{array}\right)$ commutes with $\left(\begin{array}{ll}R & 0 \\ 0 & Q\end{array}\right)$, while the diagonal blocks are of the form: $R_{i j}\left[f_{\xi} t-U\left(\mu_{i}\right) f t+f t U\left(\mu_{j}\right)\right], Q_{i j}\left[f_{\xi} t-U\left(\lambda_{i}\right) f t\right.$ $\left.+f t U\left(\lambda_{j}\right)\right]$. Since $R_{i j}$ and $Q_{i j}$ vanish unless $i=s(j)$, these terms are all proportional to $f_{\xi} t-U(s(\lambda)) f t+f t U(\lambda)$ for $\lambda=\left\{\mu_{i}, \lambda_{j}\right\}$, which vanishes by virtue of the invariance condition (3.9b). The same proof applies to the $\eta$ derivative in (3.21a). The other cases (b)-(d) are proved in a similar manner.

Finally, we shall give an alternate formulation of the constraints defining the reduction in terms of the parametrization given in Theorem (2.3) for the residues $Q_{i}$ and $R_{i}$. This result is a direct generalization of Theorem 5.3 of [8], and since the proof follows along identical lines, it will be omitted here.

Theorem 3.3. The constraints (3.14a)-(3.14d) defining the reduction of the M-matrix are equivalent to the following in the notation of Theorem 2.3:

1) If $\sigma$ is of type $\sigma_{1}$,

$$
\left.\begin{array}{l}
H_{s(i)}=f t H_{i}, \\
F_{s(i)}=(f t)^{\dagger-1} F_{i}, \\
\Gamma_{s(i) s(j)}=\Gamma_{i j}\left(c \lambda_{i}+d\right)\left(c \mu_{j}+d\right) .
\end{array}\right\}
$$

2) If $\sigma$ is of type $\sigma_{2}$,

$$
\left.\begin{array}{l}
H_{s(\bar{i})}=f t \bar{H}_{i}, \\
F_{s(\bar{i})}=(f t)^{\dagger-1} \bar{F}_{i}, \\
\Gamma_{s(\bar{i}) s(\bar{j})}=\bar{\Gamma}_{i j}\left(c \bar{\lambda}_{i}+d\right)\left(c \bar{\mu}_{j}+d\right) .
\end{array}\right\}
$$

3) If $\sigma$ is of type $\sigma_{3}$,

$$
\left.\begin{array}{l}
H_{s(i)}=f t \bar{F}_{i}, \\
F_{s(i)}=(f t)^{\dagger-1} \bar{H}_{i}, \\
\Gamma_{s(j) s(i)}^{T}=-\Gamma_{i j}\left(c \lambda_{i}+d\right)\left(c \mu_{j}+d\right) .
\end{array}\right\}
$$

4) If $\sigma$ is of type $\sigma_{4}$,

$$
\left.\begin{array}{l}
H_{s(\bar{i})}=f t F_{i}, \\
F_{s(\bar{i})}=(f t)^{\dagger-1} H_{i}, \\
\Gamma_{s(\bar{j}) s(\bar{i})}^{\dagger}=-\Gamma_{i j}\left(c \bar{\lambda}_{i}+d\right)\left(c \bar{\mu}_{j}+d\right) .
\end{array}\right\}
$$

The relations between the rectangular matrices $H_{i}, F_{i}$ should be interpreted as relating the spaces spanned by their columns, and hence may be modified by multiplication on the right by arbitrary nonsingular matrices, with $\Gamma_{i j}$ modified accordingly, consistently with Eqs. (2.29a) and (2.29b). Such a modification 
generally does not alter the residues $Q_{i}, R_{i}$, which are determined according to Theorem 2.3 by solving the linear system (2.28). However, in some cases, the constraints have no nontrivial solutions unless such a modification is made. The constraints on the $\Gamma_{i j}$ matrices are mostly redundant, since if there are no degeneracies, they follow from the definition (2.29a) and the constraints on $F_{i}$ and $H_{i}$. However, those diagonal terms with $\lambda_{i}=\mu_{i}$ are determined from Eq. (2.29b) and involve the arbitrary matrix $c(\lambda)$ of Eq. (2.22), hence the corresponding diagonal constraints are independent of those for $F_{i}$ and $H_{i}$. Furthermore, although these constraints are expressed for arbitrary $(\xi, \eta)$, it follows from Theorem 3.2 that it is sufficient that they be satisfied for the input data $\left\{f_{i}, h_{i}, c\left(\lambda_{i}\right)\right\}$ determining the value at one point, since the equations determining the $M$-matrix, and hence $\left\{F_{i}, H_{i}, \Gamma_{i j}\right\}$ then imply they are satisfied for all $(\xi, \eta)$.

\section{Examples of Reduced Systems}

To illustrate the results of the preceding sections, we shall obtain the multi-soliton solutions for two types of integrable systems by the reduction procedure: (i) the $\mathrm{SL}(n, \mathbb{R}) / \mathrm{SO}(n)$ sigma model, and (ii) Mikhailov's two-dimensional non-abelian Toda lattice [11].

\section{(i) The $\mathrm{SL}(n, \mathbb{R}) / \mathrm{SO}(n)$ Sigma Model}

This example is contained in the list of classical Riemannian symmetric spaces studied in [8]; we include it here as illustration of the general method. We start with the principal sigma model equations:

$$
A_{\eta}+B_{\xi}=0
$$

where

$$
\begin{gathered}
A=g_{\xi} g^{-1}, \quad B=g_{\eta} g^{-1}, \\
g(\xi, \eta) \in \operatorname{SL}(n, \mathbb{C}), \quad A(\xi, \eta), B(\xi, \eta) \in \operatorname{sl}(n, \mathbb{C}),
\end{gathered}
$$

which are the integrability conditions for the ZMS system:

$$
\begin{aligned}
& \psi_{\xi}=\frac{A}{1+\lambda} \psi, \\
& \psi_{\eta}=\frac{B}{1-\lambda} \psi .
\end{aligned}
$$

The reality conditions

$$
g=\bar{g}, \quad A=\bar{A}, \quad B=\bar{B},
$$

together with the involutive invariance

$$
g^{T}=g,
$$

define the $\mathrm{SL}(n, \mathbb{R}) / \mathrm{SO}(n)$ model through the Cartan immersion $[8,12]$

$$
\begin{gathered}
i: \operatorname{SL}(n, \mathbb{R}) / \mathrm{SO}(n) \rightarrow \operatorname{SL}(n, \mathbb{R}) \subset \mathrm{SL}(n, \mathbb{C}), \\
i: h \mathrm{SO}(n) \rightarrow h^{T} h \equiv g .
\end{gathered}
$$


The immersion $i$ may be locally inverted by choosing a parametrization of $\mathrm{SL}(n, \mathbb{R}) / \mathrm{SO}(n)$ by e.g. lower triangular matrices $h$ with $\operatorname{det} h=1$. The reduction group leading to conditions (4.4) and (4.5) is generated by the two transformations :

$$
\psi(\lambda) \rightarrow \bar{\psi}(\bar{\lambda})
$$

and

$$
\psi(\lambda) \rightarrow g \psi^{T-1}(1 / \lambda)
$$

where

$$
g=\psi(0), \quad \psi(\infty)=1
$$

The dressing matrix must generally include complex conjugate pairs of poles $\left\{\lambda_{i}, \bar{\lambda}_{i}\right\}$ in $\chi(\lambda)$ and $\left\{1 / \lambda_{i}, 1 / \bar{\lambda}_{i}\right\}$ in $\chi^{-1}(\lambda)$, which we parametrize

$$
\begin{gathered}
\chi(\lambda)=\mathbb{1}+\sum_{i=1}^{l}\left[\frac{Q_{i}}{\lambda-\lambda_{i}}+\frac{Q_{\bar{i}}}{\lambda-\lambda_{\bar{i}}}\right], \\
\chi^{-1}(\lambda)=\mathbb{1}+\sum_{i=1}^{l}\left[\frac{R_{\hat{i}}}{\lambda-1 / \lambda_{i}}+\frac{R_{\hat{i}}}{\lambda-1 / \bar{\lambda}_{i}}\right] .
\end{gathered}
$$

The reduction conditions following from invariance under (4.7) and (4.8) are:

$$
\begin{gathered}
\bar{\chi}(\lambda)=\chi(\bar{\lambda}), \\
\chi^{T-1}(\lambda)=\tilde{g}^{-1} \chi(1 / \lambda) g,
\end{gathered}
$$

where $\tilde{g}=\tilde{\psi}(0)$ is the new solution to the system (4.1), (4.2). According to Theorem 3.1 , the first relation (4.11) is equivalent to the following constraints on the $M$-matrix :

$$
M_{\hat{i} \bar{j}}=\bar{M}_{\hat{i} j}, \quad M_{\hat{i} \bar{j}}=\bar{M}_{\hat{i} j},
$$

which, ordering the poles as $\left\{\lambda_{1}, \ldots, \lambda_{l}, \bar{\lambda}_{1}, \ldots, \bar{\lambda}_{l}\right\},\left\{1 / \lambda_{1}, \ldots, 1 / \lambda_{l}, 1 / \bar{\lambda}_{1}, \ldots, 1 / \bar{\lambda}_{l}\right\}$ is equivalent to invariance of the space determined by $M$ under the antilinear map defined by the matrix

$$
\tilde{L}=\left(\begin{array}{cc:cc}
0 & \mathbb{1} & & \\
\mathbb{1} & 0 & & \\
\hdashline & & 0 & \mathbb{1} \\
& & \mathbb{1} & 0
\end{array}\right)
$$

In terms of the residues

$$
\left.\begin{array}{ll}
Q_{i}=X_{i} F_{i}^{\dagger}, & Q_{\bar{i}}=X_{\bar{i}} F_{\bar{i}}^{\dagger}, \\
R_{\hat{i}}=H_{\hat{i}} K_{\hat{i}}^{\dagger}, & R_{\hat{i}}=H_{\hat{i}} K_{\bar{i}}^{\dagger},
\end{array}\right\}
$$

this is equivalent, according to Theorem 3.3, to the conditions

$$
\left.\begin{array}{l}
H_{\hat{i}}=\bar{H}_{\hat{i}}, \quad F_{\bar{i}}=\bar{F}_{i}, \\
\Gamma_{\bar{i} \hat{j}}=\bar{\Gamma}_{i \hat{j}}, \quad \Gamma_{\bar{i} \hat{j}}=\bar{\Gamma}_{i \hat{j}}
\end{array}\right\}
$$


Similarly, the relation (4.12) requires the $M$-matrix to satisfy:

$$
\left.\begin{array}{rl}
M_{\hat{j} i}= & \frac{\lambda_{i}}{\lambda_{j}} g M_{\hat{i} j}^{T} g^{-1}, \\
M_{\hat{j} i}= & \frac{\lambda_{i}}{\bar{\lambda}_{j}} g M_{\hat{i} \bar{j}}^{T} g^{-1}, \\
M_{\hat{j} \bar{T}}= & \frac{\bar{\lambda}_{i}}{\lambda_{j}} g M_{\hat{i} j}^{T} g^{-1}, \\
M_{\hat{j} \bar{i}}= & \frac{\bar{\lambda}_{i}}{\bar{\lambda}_{j}} g M_{\hat{i} j}^{T} g^{-1},
\end{array}\right\}
$$

which is equivalent to the condition of total isotropy of the space determined by $M$ under the quadratic form with matrix representation:

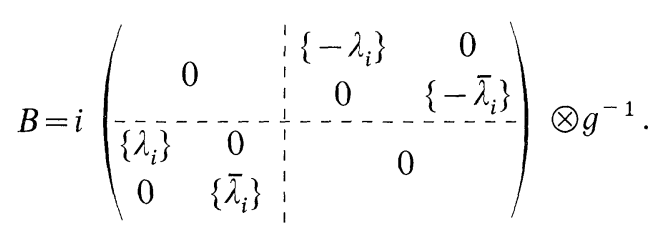

According to Theorem 3.3, this reduces to the relations

$$
\begin{gathered}
H_{\hat{i}}=g \bar{F}_{i}, \quad H_{\hat{i}}=g \bar{F}_{\bar{i}}, \\
\Gamma_{j \hat{i}}^{T}=\Gamma_{i \frac{\hat{j}}{\lambda_{i}}}, \quad \Gamma_{\hat{j} \hat{i}}^{T}=\Gamma_{\bar{i} \hat{j}} \frac{\bar{\lambda}_{i}}{\bar{\lambda}_{j}} .
\end{gathered}
$$

Thus, the solution is determined by Theorem 2.3 in terms of one rectangular matrix $f_{i}$, with $F_{i}=\psi^{\dagger-1}\left(\lambda_{i}\right) f_{i}, F_{i}, H_{\hat{i}}$, and $H_{\hat{i}}$ determined by (4.16) and (4.19) and $X_{i}, X_{\bar{i}}$, solutions to the linear system (2.28). The multi-soliton solutions may be constructed by using as input the "vacuum" solution

$$
g=\exp \left\{A_{0} \xi+B_{0} \eta\right\}, \quad \psi=\exp \left\{\frac{A_{0} \xi}{1+\lambda}+\frac{B_{0} \eta}{1-\lambda}\right\}
$$

with $A_{0}, B_{0}$ real, diagonal traceless matrices.

(ii) Non-Abelian Two-Dimensional Toda Lattice [11]

The ZMS system is:

$$
\begin{gathered}
\psi_{\xi}=\left(U_{0}+U_{1} \lambda\right) \psi, \\
\psi_{\eta}=\left(V_{1} \lambda^{-1}\right) \psi, \\
U_{0}, U_{1}, V_{1} \in \operatorname{gl}(n N, \mathbb{C}) .
\end{gathered}
$$

Following Mikhailov, the reduction group $Z_{N}$ is generated by the transformation

$$
\psi(\lambda) \rightarrow \tau \psi(q \lambda) \tau^{-1},
$$


where

$$
q=e^{2 \pi i / N}
$$

and

$$
\tau=\left(\begin{array}{cccc}
q \mathbb{1}_{n} & & & \\
& q^{2} \mathbb{1}_{n} & \\
& & \ddots & \\
& & q^{N} \mathbb{1}_{n}
\end{array}\right),
$$

giving rise to the reduced form:

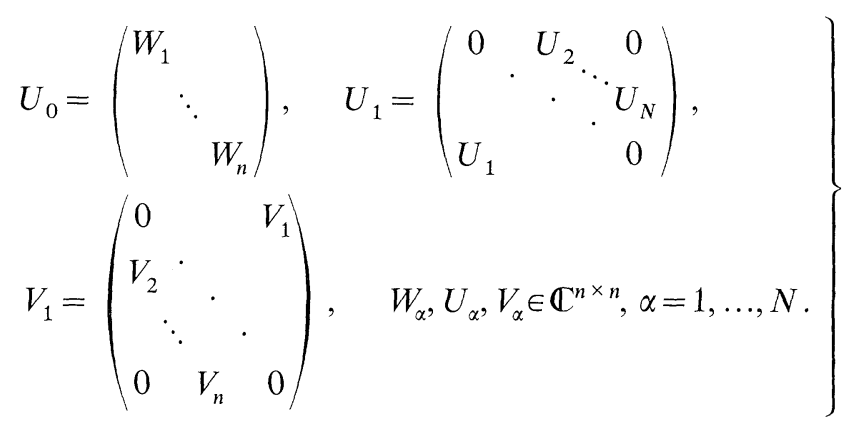

The integrability conditions for (4.21) imply in particular that the $U_{i}$ are independent of $\eta$, and the residual gauge group may be used, provided all $U_{i}$ are non-singular, to make them all equal:

$$
U_{\alpha}=U(\xi) \quad \forall \alpha .
$$

The remaining integrability conditions are:

$$
\begin{gathered}
W_{\alpha, \eta}+U V_{\alpha+1}-V_{\alpha} U=0, \\
V_{\alpha, \xi}+V_{\alpha} W_{\alpha-1}-W_{\alpha} V_{\alpha}=0, \quad \alpha=1, \ldots, N(\bmod N) .
\end{gathered}
$$

The general solution of $(4.27 \mathrm{~b})$ is of the form:

$$
V_{\alpha}=g_{\alpha} k_{\alpha}(\eta) g_{\alpha-1}^{-1}
$$

where $g_{\alpha}(\xi, \eta)$ satisfies

$$
g_{\alpha \xi}=W_{\alpha} g_{\alpha},
$$

and the $k_{\alpha}(\eta)$ are arbitrary. Assuming all $V_{\alpha}$ non-singular we may, by redefinition of $g_{\alpha}$

$$
g_{\alpha} \rightarrow g_{\alpha} l_{\alpha}(\eta)
$$

arrange that all $k_{\alpha}(\eta)$ be equal:

$$
k_{\alpha}(\eta)=V(\alpha) \quad \forall \alpha,
$$

and the remaining Eq. (4.27) becomes:

$$
\left(g_{\alpha \xi} g_{\alpha}^{-1}\right)_{\eta}+U(\xi) g_{\alpha+1} V(\eta) g_{\alpha}^{-1}-g_{\alpha} V(\eta) g_{\alpha-1}^{-1} U(\xi)=0
$$


where, without loss of generality, [because of the remaining gauge invariance and the transformations $(4.30)]$ we may assume $U(\xi), V(\eta)$ to be in Jordan normal form. Here $U(\xi), V(\eta) \in \mathrm{GL}(n, \mathbb{C})$ are arbitrary nonsingular matrix functions.

The non-abelian two-dimensional Toda lattice of Mikhailov is given by the choice

$$
U(\xi)=V(\eta)=\mathbb{1}
$$

giving the reduced form:

$$
\begin{aligned}
& U_{0}=\left(\begin{array}{ccc}
g_{1 \xi} g_{1}^{-1} & \\
& \cdot & \\
& \dot{g}_{N \xi} g_{N}^{-1}
\end{array}\right), \quad U_{1}=\left(\begin{array}{cccc}
0 & 1 & & \\
& \cdot & \cdot & \\
& & \cdot & \cdot \\
& & \cdot & 1 \\
1 & & & 0
\end{array}\right), \\
& V_{1}=\left(\begin{array}{cccc}
0 & & & g_{1} g_{N}^{-1} \\
g_{2} g_{1}^{-1} & \cdot & & \\
& \cdot & \cdot & \\
& g_{N} g_{N-1}^{-1} & 0
\end{array}\right)
\end{aligned}
$$

with integrability condition:

$$
\left(g_{\alpha \bar{\xi}} g_{\alpha}^{-1}\right)_{\eta}+g_{\alpha+1} g_{\alpha}^{-1}-g_{\alpha} g_{\alpha-1}^{-1}=0 .
$$

For the abelian case $n=1$, the choice (4.33) may be obtained from the general system by a conformal transformation. For arbitrary $n$, this seems like a further reduction, but in fact, provided the dressing matrix $\chi(\lambda)$ is such that $\chi(\infty)$ $=\chi^{-1}(\infty)=1$, this condition is preserved under the multi-Bäcklund transformation.

The only reduction condition that need be imposed is thus:

$$
\tau \chi(\lambda) \tau^{-1}=\chi\left(q^{-1} \lambda\right)
$$

which implies that $\chi(\lambda), \chi^{-1}(\lambda)$ are of the form

$$
\begin{aligned}
\chi(\lambda) & =\mathbb{1}+\sum_{i=1}^{l} \sum_{\alpha=1}^{N} \frac{Q_{i}^{\alpha}}{\lambda-\lambda_{i} q^{\alpha}}, \\
\chi^{-1}(\lambda) & =\mathbb{1}+\sum_{i=1}^{l} \sum_{\alpha=1}^{N} \frac{R_{i}^{\alpha}}{\lambda-\mu_{i} q^{\alpha}},
\end{aligned}
$$

where the various residues $\left\{Q_{i}^{\alpha}\right\},\left\{R_{i}^{\alpha}\right\}$ corresponding to a given orbit $\left\{\lambda_{i} q^{\alpha}\right\},\left\{\mu_{i} q^{\alpha}\right\}$ are related by

$$
\begin{aligned}
& Q_{i}^{\alpha}=q^{\alpha} \tau^{-\alpha} Q_{i} \tau^{\alpha}, \\
& R_{i}^{\alpha}=q^{\alpha} \tau^{-\alpha} R_{i} \tau^{\alpha}, \\
& Q_{i} \equiv Q_{i}^{N}, \quad R_{i} \equiv R_{i}^{N} .
\end{aligned}
$$

In particular, Eq. (4.36) implies that $\chi(0)$ is block diagonal,

$$
\chi(0)=\operatorname{diag}\left\{\chi_{i}(0)\right\}, \quad \chi_{i}(0, \xi, \eta) \in \operatorname{GL}(n, \mathbb{C}),
$$


and the new solution $\left\{\tilde{g}_{1}, \ldots, \tilde{g}_{N}\right\}$ is determined from the old one $\left\{g_{1}, \ldots, g_{N}\right\}$ by:

$$
g_{\alpha} \rightarrow \chi_{\alpha}(0) g_{\alpha} \equiv \tilde{g}_{\alpha} .
$$

Labelling the $n N \times n N$ blocks of the $M$-matrix corresponding to the pair of poles $\left\{\mu_{i} q^{\alpha}, \lambda_{j} q^{\beta}\right\}$ as $M_{i j}^{\alpha \beta}$, the necessary and sufficient conditions that the new solution be of the correct reduced form is, according to Theorem 3.1,

$$
M_{i j}^{\alpha+1, \beta+1}=\tau^{-1} M_{i j}^{\alpha \beta} \tau q .
$$

The representations $R$ and $Q$ of Eq. (3.16) are of the form

$$
R_{i j}^{\alpha \beta}=\delta_{i j} \delta_{\alpha, \beta-1} q^{-1 / 2}, \quad Q_{i j}^{\alpha \beta}=\delta_{i j} \delta_{\alpha, \beta-1} q^{1 / 2},
$$

which can be expressed as a tensor product

$$
R=q^{-1 / 2} \pi \otimes \mathbb{1}_{l}, \quad Q=q^{1 / 2} \pi \otimes \mathbb{1}_{l},
$$

where

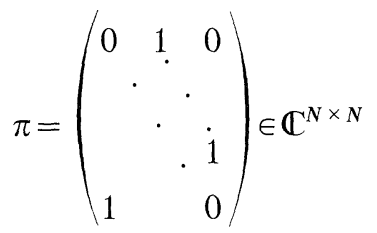

is the cyclic permutation matrix. The reduction condition (4.41) is thus equivalent to invariance of the $\ln N$-plane determined by $M$

$$
L\left[\begin{array}{c}
M \\
\mathbb{1}
\end{array}\right]=\left[\begin{array}{c}
M \\
\mathbb{1}
\end{array}\right]
$$

under the linear map with matrix representation

$$
L=\left(\begin{array}{c:c}
q^{-1 / 2} \pi \otimes \mathbb{1}_{l} & \\
\hdashline & q^{1 / 2} \pi \otimes \mathbb{1}_{l}
\end{array}\right) \otimes \tau .
$$

According to Theorem 3.3, this may be expressed equivalently in terms of the rectangular matrices $X_{i}^{\alpha}, F_{i}^{\alpha}, H_{i}^{\alpha}$, determining the residues

$$
Q_{i}^{\alpha}=X_{i}^{\alpha} F_{i}^{\dagger \alpha}
$$

where

$$
F_{i}^{\alpha}=\psi^{\dagger-1}\left(\lambda_{i} q^{\alpha}\right) f_{i}^{\alpha}, \quad H_{i}^{\alpha}=\psi\left(\mu_{i} q^{\alpha}\right) h_{i}^{\alpha}
$$

and $\left\{X_{i}^{\alpha}\right\}$ are solutions to the linear system

$$
\begin{gathered}
\sum_{i \alpha} X_{i}^{\alpha} \Gamma_{i j}^{\alpha \beta}=H_{j}^{\beta}, \\
\Gamma_{i j}^{\alpha \beta} \equiv \frac{F_{i}^{\dagger \alpha} H_{j}^{\beta}}{\lambda_{i} q^{\alpha}-\mu_{j} q^{\beta}} .
\end{gathered}
$$


The reduction conditions become:

$$
F_{i}^{\alpha}=\tau^{-\alpha} F_{i}, \quad H_{i}^{\alpha}=\tau^{-\alpha} H_{i}, \quad\left(\tau^{\dagger-1}=\tau\right),
$$

where

$$
F_{i} \equiv F_{i}^{N}, \quad H_{i} \equiv H_{i}^{N},
$$

from which it follows that:

$$
X_{i}^{\alpha}=q^{\alpha} \tau^{-\alpha} X_{i}, \quad X_{i} \equiv X_{i}^{N},
$$

and the linear system (4.49) reduces to

$$
\sum_{i \alpha} \frac{q^{\alpha} \tau^{-\alpha} X_{i} F_{i}^{\dagger} \tau^{\alpha} H_{j}}{\lambda_{i} q^{\alpha}-\mu_{j}}=H_{j} .
$$

The rectangular matrices $X_{i}, F_{i}, H_{i}$ may without loss of generality be taken as vectors, provided degenerate eigenvalues $\left\{\lambda_{i}\right\},\left\{\mu_{j}\right\}$ are allowed. Labelling components corresponding to the tensor product $\mathbb{C}^{n N}=\mathbb{C}^{N} \otimes \mathbb{C}^{n}$ as $X_{i}^{\alpha a}, F_{i}^{\alpha a}, H_{i}^{\alpha a}$, $\alpha=1, \ldots, N, a=1, \ldots, n$, Eq. (4.54) becomes :

$$
\sum_{i} X_{i}^{\beta a} W_{i j} \lambda_{i}^{\beta}=H_{j}^{\beta a} \mu_{j}^{\beta},
$$

where

$$
W_{i j} \equiv-\sum_{\gamma b} \frac{N \bar{F}_{i}^{\gamma b} H_{j}^{\gamma b}\left(\mu_{j} / \lambda_{i}\right)^{\gamma}}{\lambda_{i}\left[1-\left(\mu_{j} / \lambda_{i}\right)^{N}\right]} .
$$

The matrices $\chi_{\alpha}(0)$ in Eq. (4.40) are then given by:

$$
\chi(0)_{a b}=\mathbb{1}-N \sum_{i=1}^{l} \frac{X_{i}^{\alpha a} \bar{F}_{i}^{\alpha b}}{\lambda_{i}} .
$$

To obtain multi-soliton solutions, we identify the "vacuum" as the trivial solution

$$
g_{1}=g_{2}=\ldots=g_{N}=\mathbb{1}
$$

Solving the corresponding ZMS system:

$$
\begin{aligned}
& \psi_{\xi}^{0}=\lambda\left(\begin{array}{llll}
0 & 1 & \\
& \cdot & \cdot & \\
& \cdot & \\
& & \cdot & 1 \\
1 & & 0
\end{array}\right) \otimes \mathbb{1} \psi^{0} \\
& \psi_{\eta}^{0}=\lambda^{-1}\left(\begin{array}{lll}
0 & & 1 \\
\cdot & & \\
\cdot & . & \\
\cdot & & \\
& & \cdot \\
& 1 & 0
\end{array}\right) \otimes \mathbb{1} \psi^{0}
\end{aligned}
$$


we have, as in the abelian case [11]

$$
\psi_{\alpha a, \beta b}^{0}=\frac{\delta_{a b}}{N} \sum_{k=1}^{N} q^{(\alpha-\beta) k} \exp \left\{\lambda q^{k} \xi+\lambda^{-1} q^{-k} \eta\right\} .
$$

Substituting in Eq. (4.48) to obtain $F_{i}, H_{i}$ and solving the linear system (4.48) for $X_{i}^{\alpha a}$, the $l$-soliton solution $\left\{\tilde{g}_{1}, \ldots, \tilde{g}_{N}\right\}$ is given by the matrices $\left\{\chi_{1}(0), \ldots, \chi_{N}(0)\right\}$ of Eq. (4.57).

\section{References}

1. Zakharov, V.E., Mikhailov, A.V.: Relativistically invariant two-dimensional models of field theory which are integrable by means of the inverse problem scattering method. Zh. Eksp. Teor. Fiz. 74, 1953 (1978) [Sov. Phys. JETP 47, 1017 (1978)]

2. Zakharov, V.E., Shabat, A.B.: Integration of nonlinear equations of mathematical physics by the method of inverse scattering. Funkt. Anal. Pr. 13, 13 (1979) [English transl. Funct. Anal. Appl. 13, $166(1979)]$

3. Zakharov, V.E., Mikhailov, A.V.: On the integrability of classical spinor models in twodimensional space-time. Commun. Math. Phys. 74, 21 (1980)

4. Ablowitz, M.J., Kaup, D.J., Newell, A.C., Segur, H. : The inverse scattering transformation, Fourier analysis for nonlinear problems. Stud. Appl. Math. 53, 249 (1974)

5. Harnad, J., Saint-Aubin, Y., Shnider, S.: Superposition of solutions to Bäcklund transformations for the $\operatorname{SU}(n)$ principal sigma model. Preprint CRMA-1074; J. Math. Phys. (1984) (in press)

6. Harnad, J., Saint-Aubin, Y., Shnider, S. : Quadratic pseudopotentials for GL $(n, \mathbb{C})$ principal sigma models. Preprint CRMA-1075; Physica D (1983) (in press)

7. Saint-Aubin, Y.: Bäcklund transformations and soliton-type solutions for $\sigma$ models with values in real Grassmannian spaces. Lett. Math. Phys. 6, 441 (1983)

8. Harnad, J., Saint-Aubin, Y., Shnider, S.: Bäcklund transformations for nonlinear sigma models with values in Riemannian symmetric spaces. Commun. Math. Phys. 92, 329-367 (1984)

9. Harnad, J., Winternitz, P., Anderson, R.L.: Superposition principles for matrix Riccati equations. J. Math. Phys. 24, 1062 (1983)

10. Mikhailov, A.V.: Reduction in integrable systems. The reduction group. JETP Lett. 32, 174 (1980)

11. Mikhailov, A.V.: The reduction problem and the inverse scattering method. Physica 3D, 73 (1981)

12. Eichenherr, H., Forger, M.: On the dual symmetry of the non-linear sigma models. Nucl. Phys. B 155, 381 (1979); More about non-linear sigma models on symmetric spaces. Nucl. Phys. B 164, 528 (1980)

Communicated by G. Mack

Received July 12, 1983 